\title{
Strong Insertion of a Contra-continuous Function between Two Comparable Contra-precontinuous (Contra-semi-continuous) of Real- valued Functions
}

\author{
Majid Mirmiran $^{1}$ and Binesh Naderi ${ }^{2}$ \\ ${ }^{1}$ Department of Mathematics, University of Isfahan, Isfahan 81746-73441, Iran \\ e-mail:mirmir@sci.ui.ac.ir \\ ${ }^{2}$ Department of General Courses, School of Management and Medical Information Sciences, \\ Isfahan University of Medical Sciences, Isfahan, Iran \\ e-mail: naderi@mng.mui.ac.ir
}

\begin{abstract}
Necessary and sufficient conditions in terms of lower cut sets are given for the strong insertion of a contra-continuous function between two comparable real-valued functions on such topological spaces that kernel of sets are open.
\end{abstract}

\section{Introduction}

The concept of a preopen set in a topological space was introduced by Corson and Michael in 1964 [4]. A subset $A$ of a topological space $(X, \tau)$ is called preopen or locally dense or nearly open if $A \subseteq \operatorname{Int}(C l(A))$. A set $A$ is called preclosed if its complement is preopen or equivalently if $\operatorname{Cl}(\operatorname{Int}(A)) \subseteq A$. The term, preopen, was used for the first time by Mashhour et al. [21], while the concept of a, locally dense, set was introduced by Corson and Michael [4].

Received: December 26, 2019; Accepted: February 20, 2020

2010 Mathematics Subject Classification: Primary 54C08, 54C10, 54C50; Secondary 26A15, 54C30.

Keywords and phrases: insertion, strong binary relation, semi-open set, preopen set, contra-continuous function, lower cut set.

Copyright () 2020 Majid Mirmiran and Binesh Naderi. This is an open access article distributed under the Creative Commons Attribution License, which permits unrestricted use, distribution, and reproduction in any medium, provided the original work is properly cited. 
The concept of a semi-open set in a topological space was introduced by Levine in 1963 [18]. A subset $A$ of a topological space $(X, \tau)$ is called semi-open [10] if $A \subseteq C l(\operatorname{Int}(A))$. A set $A$ is called semi-closed if its complement is semi-open or equivalently if $\operatorname{Int}(C l(A)) \subseteq A$.

A generalized class of closed sets was considered by Maki in [20]. He investigated the sets that can be represented as union of closed sets and called them $V$-sets. Complements of $V$-sets, i.e., sets that are intersection of open sets are called $\Lambda$-sets [20].

Recall that a real-valued function $f$ defined on a topological space $X$ is called $A$ continuous [28] if the preimage of every open subset of $\mathbb{R}$ belongs to $A$, where $A$ is a collection of subsets of $X$. Most of the definitions of function used throughout this paper are consequences of the definition of $A$-continuity. However, for unknown concepts the reader may refer to $[5,11]$. In the recent literature many topologists had focused their research in the direction of investigating different types of generalized continuity.

Dontchev in [6] introduced a new class of mappings called contra-continuity. A good number of researchers have also initiated different types of contra-continuous like mappings in the papers $[1,3,8,9,10,12,13,26]$.

Hence, a real-valued function $f$ defined on a topological space $X$ is called contracontinuous (resp. contra-semi-continuous, contra-precontinuous) if the preimage of every open subset of $\mathbb{R}$ is closed (resp. semi-closed, pre-closed) in $X$ [6].

Results of Katětov [14, 15] concerning binary relations and the concept of an indefinite lower cut set for a real-valued function, which is due to Brooks [2], are used in order to give a necessary and sufficient conditions for the insertion of a contracontinuous function between two comparable real-valued functions on such topological spaces that $\Lambda$-sets or kernel of sets are open [20].

If $g$ and $f$ are real-valued functions defined on a space $X$, we write $g \leq f$ in case $g(x) \leq f(x)$ for all $x$ in $X$.

The following definitions are modifications of conditions considered in [16].

A property $P$ defined relative to a real-valued function on a topological space is a $c c$ property provided that any constant function has property $P$ and provided that the sum of a function with property $P$ and any contra-continuous function also has property $P$. If $P_{1}$ 
and $P_{2}$ are $c c$-properties, the following terminology is used: (i) A space $X$ has the weak cc-insertion property for $\left(P_{1}, P_{2}\right)$ if and only if for any functions $g$ and $f$ on $X$ such that $g \leq f, g$ has property $P_{1}$ and $f$ has property $P_{2}$, then there exists a contra-continuous function $h$ such that $g \leq h \leq f$. (ii) A space $X$ has the strong cc-insertion property for $\left(P_{1}, P_{2}\right)$ if and only if for any functions $g$ and $f$ on $X$ such that $g \leq f, g$ has property $P_{1}$ and $f$ has property $P_{2}$, then there exists a contra-continuous function $h$ such that $g \leq h \leq f$ and if $g(x)<f(x)$ for any $x$ in $X$, then $g(x)<h(x)<f(x)$.

In this paper, for a topological space whose $\Lambda$-sets or kernel of sets are open, is given a sufficient condition for the weak $c c$-insertion property. Also for a space with the weak $c c$-insertion property, we give necessary and sufficient conditions for the space to have the strong $c c$-insertion property. Several insertion theorems are obtained as corollaries of these results. In addition, the insertion and strong insertion of a contra- $\alpha$-continuous function and insertion of a contra-continuous function between two comparable realvalued functions has also recently considered by the authors in [22, 23, 24].

\section{The Main Result}

Before giving a sufficient condition for insertability of a contra-continuous function, the necessary definitions and terminology are stated.

The abbreviations $c c, \quad c p c$ and $c s c$ are used for contra-continuous, contraprecontinuous and contra-semi-continuous, respectively.

Definition 2.1. Let $A$ be a subset of a topological space $(X, \tau)$. We define the subsets $A^{\Lambda}$ and $A^{V}$ as follows:

$$
A^{\Lambda}=\bigcap\{O: O \supseteq A, O \in(X, \tau)\} \text { and } A^{V}=\bigcup\left\{F: F \subseteq A, F^{c} \in(X, \tau)\right\} .
$$

In [7, 19, 25], $A^{\Lambda}$ is called the kernel of $A$.

The family of all preopen, preclosed, semi-open and semi-closed will be denoted by $p O(X, \tau), p C(X, \tau), s O(X, \tau)$, and $s C(X, \tau)$, respectively.

We define the subsets $p\left(A^{\Lambda}\right), p\left(A^{V}\right), s\left(A^{\Lambda}\right)$ and $s\left(A^{V}\right)$ as follows:

$$
p\left(A^{\Lambda}\right)=\bigcap\{O: O \supseteq A, O \in p O(X, \tau)\},
$$




$$
\begin{aligned}
& p\left(A^{V}\right)=\bigcup\{F: F \subseteq A, F \in p C(X, \tau)\}, \\
& s\left(A^{\Lambda}\right)=\bigcap\{O: O \supseteq A, O \in s O(X, \tau)\}
\end{aligned}
$$

and

$$
s\left(A^{V}\right)=\bigcup\{F: F \subseteq A, F \in s C(X, \tau)\} .
$$

$p\left(A^{\Lambda}\right)$ (resp. $\left.s\left(A^{\Lambda}\right)\right)$ is called the prekernel (resp. semi-kernel) of $A$.

The following first two definitions are modifications of conditions considered in [14, $15]$.

Definition 2.2. If $\rho$ is a binary relation in a set $S$, then $\bar{\rho}$ is defined as follows: $x \bar{\rho} y$ if and only if $y \rho v$ implies $x \rho v$ and $u \rho x$ implies $u \rho y$ for $u$ and $v$ in $S$.

Definition 2.3. A binary relation $\rho$ in the power set $P(X)$ of a topological space $X$ is called a strong binary relation in $P(X)$ in case $\rho$ satisfies each of the following conditions:

(1) If $A_{i} \rho B_{j}$ for any $i \in\{1, \ldots, m\}$ and for any $j \in\{1, \ldots, n\}$, then there exists a set $C$ in $P(X)$ such that $A_{i} \rho C$ and $C \rho B_{j}$ for any $i \in\{1, \ldots, m\}$ and any $j \in\{1, \ldots, n\}$.

(2) If $A \subseteq B$, then $A \bar{\rho} B$.

(3) If $A \rho B$, then $A^{\Lambda} \subseteq B$ and $A \subseteq B^{V}$.

The concept of a lower indefinite cut set for a real-valued function was defined by Brooks [2] as follows:

Definition 2.4. If $f$ is a real-valued function defined on a space $X$ and if $\{x \in X: f(x)<\ell\} \subseteq A(f, \ell) \subseteq\{x \in X: f(x) \leq \ell\}$ for a real number $\ell$, then $A(f, \ell)$ is called a lower indefinite cut set in the domain of $f$ at the level $\ell$.

We now give the following main result:

Theorem 2.1. Let $g$ and $f$ be real-valued functions on the topological space $X$, in which kernel sets are open, with $g \leq f$. If there exists a strong binary relation $\rho$ on the 
power set of $X$ and if there exist lower indefinite cut sets $A(f, t)$ and $A(g, t)$ in the domain of $f$ and $g$ at the level $t$ for each rational number $t$ such that if $t_{1}<t_{2}$, then $A\left(f, t_{1}\right) \rho A\left(g, t_{2}\right)$, then there exists a contra-continuous function $h$ defined on $X$ such that $g \leq h \leq f$.

Proof. Let $g$ and $f$ be real-valued functions defined on the $X$ such that $g \leq f$. By hypothesis there exists a strong binary relation $\rho$ on the power set of $X$ and there exist lower indefinite cut sets $A(f, t)$ and $A(g, t)$ in the domain of $f$ and $g$ at the level $t$ for each rational number $t$ such that if $t_{1}<t_{2}$, then $A\left(f, t_{1}\right) \rho A\left(g, t_{2}\right)$.

Define functions $F$ and $G$ mapping the rational numbers $\mathbb{Q}$ into the power set of $X$ by $F(t)=A(f, t)$ and $G(t)=A(g, t)$. If $t_{1}$ and $t_{2}$ are any elements of $\mathbb{Q}$ with $t_{1}<t_{2}$, then $F\left(t_{1}\right) \bar{\rho} F\left(t_{2}\right), G\left(t_{1}\right) \bar{\rho} G\left(t_{2}\right)$, and $F\left(t_{1}\right) \rho G\left(t_{2}\right)$. By Lemmas 1 and 2 of [15] it follows that there exists a function $H$ mapping $\mathbb{Q}$ into the power set of $X$ such that if $t_{1}$ and $t_{2}$ are any rational numbers with $t_{1}<t_{2}$, then $F\left(t_{1}\right) \rho H\left(t_{2}\right), H\left(t_{1}\right) \rho H\left(t_{2}\right)$ and $H\left(t_{1}\right) \rho G\left(t_{2}\right)$.

For any $x$ in $X$, let $h(x)=\inf \{t \in \mathbb{Q}: x \in H(t)\}$.

We first verify that $g \leq h \leq f$ : If $x$ is in $H(t)$, then $x$ is in $G\left(t^{\prime}\right)$ for any $t^{\prime}>t$; since $x$ is in $G\left(t^{\prime}\right)=A\left(g, t^{\prime}\right)$ implies that $g(x) \leq t^{\prime}$, it follows that $g(x) \leq t$. Hence $g \leq h$. If $x$ is not in $H(t)$, then $x$ is not in $F\left(t^{\prime}\right)$ for any $t^{\prime}<t$; since $x$ is not in $F\left(t^{\prime}\right)=A\left(f, t^{\prime}\right)$ implies that $f(x)>t^{\prime}$, it follows that $f(x) \geq t$. Hence $h \leq f$.

Also, for any rational numbers $t_{1}$ and $t_{2}$ with $t_{1}<t_{2}$, we have $h^{-1}\left(t_{1}, t_{2}\right)=$ $H\left(t_{2}\right)^{V} \backslash H\left(t_{1}\right)^{\Lambda}$. Hence $h^{-1}\left(t_{1}, t_{2}\right)$ is closed in $X$, i.e., $h$ is a contra-continuous function on $X$.

The above proof used the technique of Theorem 1 in [14].

If a space has the strong $c c$-insertion property for $\left(P_{1}, P_{2}\right)$, then it has the weak $c c$-insertion property for $\left(P_{1}, P_{2}\right)$. The following result uses lower cut sets and gives a necessary and sufficient condition for a space satisfies that weak $c c$-insertion property to satisfy the strong $c c$-insertion property. 
Theorem 2.2. Let $P_{1}$ and $P_{2}$ be cc-property and $X$ be a space that satisfies the weak cc-insertion property for $\left(P_{1}, P_{2}\right)$. Also assume that $g$ and $f$ are functions on $X$ such that $g \leq f, g$ has property $P_{1}$ and $f$ has property $P_{2}$. The space $X$ has the strong $c c$ insertion property for $\left(P_{1}, P_{2}\right)$ if and only if there exist lower cut sets $A\left(f-g, 2^{-n}\right)$ and there exists a sequence $\left\{F_{n}\right\}$ of subsets of $X$ such that (i) for each $n, F_{n}$ and $A\left(f-g, 2^{-n}\right)$ are completely separated by contra-continuous functions, and (ii) $\{x \in X:(f-g)(x)>0\}=\bigcup_{n=1}^{\infty} F_{n}$.

Proof. Suppose that there is a sequence $\left(A\left(f-g, 2^{-n}\right)\right)$ of lower cut sets for $f-g$ and suppose that there is a sequence $\left(F_{n}\right)$ of subsets of $X$ such that

$$
\{x \in X:(f-g)(x)>0\}=\bigcup_{n=1}^{\infty} F_{n}
$$

and such that for each $n$, there exists a contra-continuous function $k_{n}$ on $X$ into $\left[0,2^{-n}\right]$ with $k_{n}=2^{-n}$ on $F_{n}$ and $k_{n}=0$ on $A\left(f-g, 2^{-n}\right)$. The function $k$ from $X$ into $[0,1 / 4]$ which is defined by

$$
k(x)=1 / 4 \sum_{n=1}^{\infty} k_{n}(x)
$$

is a contra-continuous function by the Cauchy condition and the properties of contracontinuous functions, (1) $k^{-1}(0)=\{x \in X:(f-g)(x)=0\}$ and $(2)$ if $(f-g)(x)>0$, then $k(x)<(f-g)(x):$ In order to verify (1), observe that if $(f-g)(x)=0$, then $x \in A\left(f-g, 2^{-n}\right)$ for each $n$ and hence $k_{n}(x)=0$ for each $n$. Thus $k(x)=0$. Conversely, if $(f-g)(x)>0$, then there exists an $n$ such that $x \in F_{n}$ and hence $k_{n}(x)=2^{-n}$. Thus $k(x) \neq 0$ and this verifies (1). Next, in order to establish (2), note that

$$
\{x \in X:(f-g)(x)=0\}=\bigcap_{n=1}^{\infty} A\left(f-g, 2^{-n}\right)
$$


and that $\left(A\left(f-g, 2^{-n}\right)\right)$ is a decreasing sequence. Thus if $(f-g)(x)>0$, then either $x \notin A(f-g, 1 / 2)$ or there exists a smallest $n$ such that $x \notin A\left(f-g, 2^{-n}\right)$ and $x \in A\left(f-g, 2^{-j}\right)$ for $j=1, \ldots, n-1$.

In the former case,

$$
k(x)=1 / 4 \sum_{n=1}^{\infty} k_{n}(x) \leq 1 / 4 \sum_{n=1}^{\infty} 2^{-n}<1 / 2 \leq(f-g)(x),
$$

and in the latter,

$$
k(x)=1 / 4 \sum_{j=n}^{\infty} k_{j}(x) \leq 1 / 4 \sum_{j=n}^{\infty} 2^{-j}<2^{-n} \leq(f-g)(x) .
$$

Thus $0 \leq k \leq f-g$ and if $(f-g)(x)>0$, then $(f-g)(x)>k(x)>0$. Let $g_{1}=g+(1 / 4) k$ and $f_{1}=f-(1 / 4) k$. Then $g \leq g_{1} \leq f_{1} \leq f$ and if $g(x)<f(x)$, then

$$
g(x)<g_{1}(x)<f_{1}(x)<f(x) .
$$

Since $P_{1}$ and $P_{2}$ are $c c$-properties, then $g_{1}$ has property $P_{1}$ and $f_{1}$ has property $P_{2}$. Since by hypothesis $X$ has the weak $c c$-insertion property for $\left(P_{1}, P_{2}\right)$, then there exists a contra-continuous function $h$ such that $g_{1} \leq h \leq f_{1}$. Thus $g \leq h \leq f$ and if $g(x)<f(x)$, then $g(x)<h(x)<f(x)$. Therefore $X$ has the strong $c c$-insertion property for $\left(P_{1}, P_{2}\right)$. (The technique of this proof is by Lane [16].)

Conversely, assume that $X$ satisfies the strong $c c$-insertion for $\left(P_{1}, P_{2}\right)$. Let $g$ and $f$ be functions on $X$ satisfying $P_{1}$ and $P_{2}$ respectively such that $g \leq f$. Thus there exists a contra-continuous function $h$ such that $g \leq h \leq f$ and such that if $g(x)<f(x)$ for any $x$ in $X$, then $g(x)<h(x)<f(x)$. We follow an idea contained in Powderly [27]. Now consider the functions 0 and $f-h$. 0 satisfies property $P_{1}$ and $f-h$ satisfies property $P_{2}$. Thus there exists a contra-continuous function $h_{1}$ such that $0 \leq h_{1} \leq f-h$ and if $0<(f-h)(x)$ for any $x$ in $X$, then $0<h_{1}(x)<(f-h)(x)$. We next show that

$$
\{x \in X:(f-g)(x)>0\}=\left\{x \in X: h_{1}(x)>0\right\} .
$$


If $x$ is such that $(f-g)(x)>0$, then $g(x)<f(x)$. Therefore $g(x)<h(x)<f(x)$. Thus $f(x)-h(x)>0$ or $(f-h)(x)>0$. Hence $h_{1}(x)>0$. On the other hand, if $h_{1}(x)>0$, then since $(f-h) \geq h_{1}$ and $f-g \geq f-h$, therefore $(f-g)(x)>0$. For each $n$, let

$$
\begin{gathered}
A\left(f-g, 2^{-n}\right)=\left\{x \in X:(f-g)(x) \leq 2^{-n}\right\}, \\
F_{n}=\left\{x \in X: h_{1}(x) \geq 2^{-n+1}\right\}
\end{gathered}
$$

and

$$
k_{n}=\sup \left\{\inf \left\{h_{1}, 2^{-n+1}\right\}, 2^{-n}\right\}-2^{-n} .
$$

Since $\{x \in X:(f-g)(x)>0\}=\left\{x \in X: h_{1}(x)>0\right\}$, it follows that

$$
\{x \in X:(f-g)(x)>0\}=\bigcup_{n=1}^{\infty} F_{n} .
$$

We next show that $k_{n}$ is a contra-continuous function which completely separates $F_{n}$ and $A\left(f-g, 2^{-n}\right)$. From its definition and by the properties of contra-continuous functions, it is clear that $k_{n}$ is a contra-continuous function. Let $x \in F_{n}$. Then, from the definition of $k_{n}, k_{n}(x)=2^{-n}$. If $x \in A\left(f-g, 2^{-n}\right)$, then since $h_{1} \leq f-h \leq f-g$, $h_{1}(x) \leq 2^{-n}$. Thus $k_{n}(x)=0$, according to the definition of $k_{n}$. Hence $k_{n}$ completely separates $F_{n}$ and $A\left(f-g, 2^{-n}\right)$.

Theorem 2.3. Let $P_{1}$ and $P_{2}$ be cc-properties and assume that the space $X$ satisfied the weak cc-insertion property for $\left(P_{1}, P_{2}\right)$. The space $X$ satisfies the strong cc-insertion property for $\left(P_{1}, P_{2}\right)$ if and only if $X$ satisfies the strong cc-insertion property for $\left(P_{1}, c c\right)$ and for $\left(c c, P_{2}\right)$.

Proof. Assume that $X$ satisfies the strong $c c$-insertion property for $\left(P_{1}, c c\right)$ and for $\left(c c, P_{2}\right)$. If $g$ and $f$ are functions on $X$ such that $g \leq f, g$ satisfies property $P_{1}$, and $f$ satisfies property $P_{2}$, then since $X$ satisfies the weak $c c$-insertion property for $\left(P_{1}, P_{2}\right)$ there is a contra-continuous function $k$ such that $g \leq k \leq f$. Also, by hypothesis there exist contra-continuous functions $h_{1}$ and $h_{2}$ such that $g \leq h_{1} \leq k$ and if $g(x)<k(x)$, 
then $g(x)<h_{1}(x)<k(x)$ and such that $k \leq h_{2} \leq f$ and if $k(x)<f(x)$, then $k(x)<h_{2}(x)<f(x)$. If a function $h$ is defined by $h(x)=\left(h_{2}(x)+h_{1}(x)\right) / 2$, then $h$ is a contra-continuous function, $g \leq h \leq f$, and if $g(x)<f(x)$, then $g(x)<h(x)<f(x)$. Hence $X$ satisfies the strong $c c$-insertion property for $\left(P_{1}, P_{2}\right)$.

The converse is obvious since any contra-continuous function must satisfy both properties $P_{1}$ and $P_{2}$. (The technique of this proof is by Lane [17].)

\section{Applications}

Before stating the consequences of Theorems 2.1,2.2, and 2.3 we suppose that $X$ is a topological space whose kernel sets are open.

Corollary 3.1. If for each pair of disjoint preopen (resp. semi-open) sets $G_{1}, G_{2}$ of $X$, there exist closed sets $F_{1}$ and $F_{2}$ of $X$ such that $G_{1} \subseteq F_{1}, G_{2} \subseteq F_{2}$ and $F_{1} \cap F_{2}=\varnothing$, then $X$ has the weak cc-insertion property for (cpc, cpc) (resp. $(c s c, c s c))$.

Proof. Let $g$ and $f$ be real-valued functions defined on $X$, such that $f$ and $g$ are cpc (resp. csc), and $g \leq f$. If a binary relation $\rho$ is defined by $A \rho B$ in case $p\left(A^{\Lambda}\right) \subseteq p\left(B^{V}\right)$ (resp. $\left.s\left(A^{\Lambda}\right) \subseteq s\left(B^{V}\right)\right)$, then by hypothesis $\rho$ is a strong binary relation in the power set of $X$. If $t_{1}$ and $t_{2}$ are any elements of $Q$ with $t_{1}<t_{2}$, then

$$
A\left(f, t_{1}\right) \subseteq\left\{x \in X: f(x) \leq t_{1}\right\} \subseteq\left\{x \in X: g(x)<t_{2}\right\} \subseteq A\left(g, t_{2}\right) ;
$$

since $\left\{x \in X: f(x) \leq t_{1}\right\}$ is a preopen (resp. semi-open) set and since $\left\{x \in X: g(x)<t_{2}\right\}$ is a preclosed (resp. semi-closed) set, it follows that $p\left(A\left(f, t_{1}\right)^{\Lambda}\right) \subseteq p\left(A\left(g, t_{2}\right)^{V}\right) \quad$ (resp. $s\left(A\left(f, t_{1}\right)^{\Lambda}\right) \subseteq s\left(A\left(g, t_{2}\right)^{V}\right)$. Hence $t_{1}<t_{2}$ implies that $A\left(f, t_{1}\right) \rho A\left(g, t_{2}\right)$. The proof follows from Theorem 2.1.

Corollary 3.2. If for each pair of disjoint preopen (resp. semi-open) sets $G_{1}, G_{2}$, there exist closed sets $F_{1}$ and $F_{2}$ such that $G_{1} \subseteq F_{1}, G_{2} \subseteq F_{2}$ and $F_{1} \cap F_{2}=\varnothing$, then every contra-precontinuous (resp. contra-semi-continuous) function is contracontinuous. 
Proof. Let $f$ be a real-valued contra-precontinuous (resp. contra-semi-continuous) function defined on $X$. Set $g=f$, then by Corollary 3.1, there exists a contracontinuous function $h$ such that $g=h=f$.

Corollary 3.3. If for each pair of disjoint preopen (resp. semi-open) sets $G_{1}, G_{2}$ of $X$, there exist closed sets $F_{1}$ and $F_{2}$ of $X$ such that $G_{1} \subseteq F_{1}, G_{2} \subseteq F_{2}$ and $F_{1} \cap F_{2}=\varnothing$, then $X$ has the cc-insertion property for $(c p c, c p c)($ resp. $(c s c, c s c))$.

Proof. Let $g$ and $f$ be real-valued functions defined on the $X$, such that $f$ and $g$ are cpc (resp. csc), and $g<f$. Set $h=(f+g) / 2$, thus $g \leq h \leq f$ and if $g(x)<f(x)$ for any $x$ in $X$, then $g(x)<h(x)<f(x)$. Also, by Corollary 3.2, since $g$ and $f$ are contracontinuous functions hence $h$ is a contra-continuous function.

Corollary 3.4. If for each pair of disjoint subsets $G_{1}, G_{2}$ of $X$, such that $G_{1}$ is preopen and $G_{2}$ is semi-open, there exist closed subsets $F_{1}$ and $F_{2}$ of $X$ such that $G_{1} \subseteq F_{1}, G_{2} \subseteq F_{2}$ and $F_{1} \cap F_{2}=\varnothing$, then $X$ have the weak cc-insertion property for $(c p c, c s c)$ and $(c s c, c p c)$.

Proof. Let $g$ and $f$ be real-valued functions defined on $X$, such that $g$ is $c p c$ (resp. csc) and $f$ is $\csc$ (resp. $c p c$ ), with $g \leq f$. If a binary relation $\rho$ is defined by $A \rho B$ in case $s\left(A^{\Lambda}\right) \subseteq p\left(B^{V}\right)$ (resp. $p\left(A^{\Lambda}\right) \subseteq s\left(B^{V}\right)$ ), then by hypothesis $\rho$ is a strong binary relation in the power set of $X$. If $t_{1}$ and $t_{2}$ are any elements of $Q$ with $t_{1}<t_{2}$, then

$$
A\left(f, t_{1}\right) \subseteq\left\{x \in X: f(x) \leq t_{1}\right\} \subseteq\left\{x \in X: g(x)<t_{2}\right\} \subseteq A\left(g, t_{2}\right)
$$

since $\left\{x \in X: f(x) \leq t_{1}\right\} \quad$ is a semi-open (resp. preopen) set and since $\left\{x \in X: g(x)<t_{2}\right\}$ is a preclosed (resp. semi-closed) set, it follows that $s\left(A\left(f, t_{1}\right)^{\Lambda}\right) \subseteq p\left(A\left(g, t_{2}\right)^{V}\right)$ (resp. $\left.p\left(A\left(f, t_{1}\right)^{\Lambda}\right) \subseteq s\left(A\left(g, t_{2}\right)^{V}\right)\right)$. Hence $t_{1}<t_{2}$, implies that $A\left(f, t_{1}\right) \rho A\left(g, t_{2}\right)$. The proof follows from Theorem 2.1.

Before stating consequences of Theorems 2.2, 2.3 we state and prove the necessary lemmas. 
Lemma 3.1. The following conditions on the space $X$ are equivalent:

(i) For each pair of disjoint subsets $G_{1}, G_{2}$ of $X$, such that $G_{1}$ is preopen and $G_{2}$ is semi-open, there exist closed subsets $F_{1}, F_{2}$ of $X$ such that $G_{1} \subseteq F_{1}, G_{2} \subseteq F_{2}$ and $F_{1} \cap F_{2}=\varnothing$.

(ii) If $G$ is a semi-open (resp. preopen) subset of $X$ which is contained in a preclosed (resp. semi-closed) subset $F$ of $X$, then there exists a closed subset $H$ of $X$ such that $G \subseteq H \subseteq H^{\Lambda} \subseteq F$.

Proof. (i) $\Rightarrow$ (ii) Suppose that $G \subseteq F$, where $G$ and $F$ are semi-open (resp. preopen) and preclosed (resp. semi-closed) subsets of $X$, respectively. Hence, $F^{c}$ is a preopen (resp. semi-open) and $G \cap F^{c}=\varnothing$.

By (i) there exists two disjoint closed subsets $F_{1}, F_{2}$ such that $G \subseteq F_{1}$ and $F^{c} \subseteq F_{2}$. But

$$
F^{c} \subseteq F_{2} \Rightarrow F_{2}^{c} \subseteq F
$$

and

$$
F_{1} \cap F_{2}=\varnothing \Rightarrow F_{1} \subseteq F_{2}^{c}
$$

hence

$$
G \subseteq F_{1} \subseteq F_{2}^{c} \subseteq F
$$

and since $F_{2}^{c}$ is an open subset containing $F_{1}$, we conclude that $F_{1}^{\Lambda} \subseteq F_{2}^{c}$, i.e.,

$$
G \subseteq F_{1} \subseteq F_{1}^{\Lambda} \subseteq F
$$

By setting $H=F_{1}$, condition(ii) holds.

(ii) $\Rightarrow$ (i) Suppose that $G_{1}, G_{2}$ are two disjoint subsets of $X$, such that $G_{1}$ is preopen and $G_{2}$ is semi-open.

This implies that $G_{2} \subseteq G_{1}^{c}$ and $G_{1}^{c}$ is a preclosed subset of $X$. Hence by (ii) there exists a closed set $H$ such that $G_{2} \subseteq H \subseteq H^{\Lambda} \subseteq G_{1}^{c}$. 
But

$$
H \subseteq H^{\Lambda} \Rightarrow H \cap\left(H^{\Lambda}\right)^{c}=\varnothing
$$

and

$$
H^{\Lambda} \subseteq G_{1}^{c} \Rightarrow G_{1} \subseteq\left(H^{\Lambda}\right)^{c}
$$

Furthermore, $\left(H^{\Lambda}\right)^{c}$ is a closed subset of $X$. Hence $G_{2} \subseteq H, G_{1} \subseteq\left(H^{\Lambda}\right)^{c}$ and $H \cap\left(H^{\Lambda}\right)^{c}=\varnothing$. This means that condition (i) holds.

Lemma 3.2. Suppose that $X$ is a topological space. If each pair of disjoint subsets $G_{1}, G_{2}$ of $X$, where $G_{1}$ is preopen and $G_{2}$ is semi-open, can be separated by closed subsets of $X$, then there exists a contra-continuous function $h: X \rightarrow[0,1]$ such that $h\left(G_{2}\right)=\{0\}$ and $h\left(G_{1}\right)=\{1\}$.

Proof. Suppose $G_{1}$ and $G_{2}$ are two disjoint subsets of $X$, where $G_{1}$ is preopen and $G_{2}$ is semi-open. Since $G_{1} \cap G_{2}=\varnothing$, hence $G_{2} \subseteq G_{1}^{c}$. In particular, since $G_{1}^{c}$ is a preclosed subset of $X$ containing the semi-open subset $G_{2}$ of $X$, by Lemma 3.1, there exists a closed subset $H_{1 / 2}$ such that

$$
G_{2} \subseteq H_{1 / 2} \subseteq H_{1 / 2}^{\Lambda} \subseteq G_{1}^{c}
$$

Note that $H_{1 / 2}$ is also a preclosed subset of $X$ and contains $G_{2}$, and $G_{1}^{c}$ is a preclosed subset of $X$ and contains the semi-open subset $H_{1 / 2}^{\Lambda}$ of $X$. Hence, by Lemma 3.1, there exists closed subsets $H_{1 / 4}$ and $H_{3 / 4}$ such that

$$
G_{2} \subseteq H_{1 / 4} \subseteq H_{1 / 4}^{\Lambda} \subseteq H_{1 / 2} \subseteq H_{1 / 2}^{\Lambda} \subseteq H_{3 / 4} \subseteq H_{3 / 4}^{\Lambda} \subseteq G_{1}^{c}
$$

By continuing this method for every $t \in D$, where $D \subseteq[0,1]$ is the set of rational numbers that their denominators are exponents of 2, we obtain closed subsets $H_{t}$ with the property that if $t_{1}, t_{2} \in D$ and $t_{1}<t_{2}$, then $H_{t_{1}} \subseteq H_{t_{2}}$. We define the function $h$ on $X$ by $h(x)=\inf \left\{t: x \in H_{t}\right\}$ for $x \notin G_{1}$ and $h(x)=1$ for $x \in G_{1}$. 
Note that for every $x \in X, 0 \leq h(x) \leq 1$, i.e., $h$ maps $X$ into [0,1]. Also, we note that for any $t \in D, G_{2} \subseteq H_{t}$; hence $h\left(G_{2}\right)=\{0\}$. Furthermore, by definition, $h\left(G_{1}\right)=\{1\}$. It remains only to prove that $h$ is a contra-continuous function on $X$. For every $\alpha \in \mathbb{R}$, we have if $\alpha \leq 0$, then $\{x \in X: h(x)<\alpha\}=\varnothing$ and if $0<\alpha$, then $\{x \in X: h(x)<\alpha\}=\bigcup\left\{H_{t}: t<\alpha\right\}$, hence, they are closed subsets of $X$. Similarly, if $\alpha<0$, then $\{x \in X: h(x)>\alpha\}=X \quad$ and if $0 \leq \alpha$, then $\{x \in X: h(x)>\alpha\}=$ $\bigcup\left\{\left(H_{t}^{\Lambda}\right)^{c}: t>\alpha\right\}$ hence, every of them is a closed subset. Consequently $h$ is a contracontinuous function.

Lemma 3.3. Suppose that $X$ is a topological space. If each pair of disjoint subsets $G_{1}, G_{2}$ of $X$, where $G_{1}$ is preopen and $G_{2}$ is semi-open, can separate by closed subsets of $X$, and $G_{1}$ (resp. $G_{2}$ ) is a closed subsets of $X$, then there exists a contra-continuous function $h: X \rightarrow[0,1]$ such that, $h^{-1}(0)=G_{1}\left(\right.$ resp. $\left.h^{-1}(0)=G_{2}\right)$ and $h\left(G_{2}\right)=\{1\}$ (resp. $h\left(G_{1}\right)=\{1\}$ ).

Proof. Suppose that $G_{1}$ (resp. $G_{2}$ ) is a closed subset of $X$. By Lemma 3.2, there exists a contra-continuous function $h: X \rightarrow[0,1]$ such that, $h\left(G_{1}\right)=\{0\} \quad$ (resp. $\left.h\left(G_{2}\right)=\{0\}\right)$ and $h\left(X \backslash G_{1}\right)=\{1\}$ (resp. $h\left(X \backslash G_{2}\right)=\{1\}$ ). Hence, $h^{-1}(0)=G_{1}$ (resp. $\left.h^{-1}(0)=G_{2}\right)$ and since $G_{2} \subseteq X \backslash G_{1}$ (resp. $\left.G_{1} \subseteq X \backslash G_{2}\right)$, therefore $h\left(G_{2}\right)=\{1\}$ (resp. $\left.h\left(G_{1}\right)=\{1\}\right)$.

Lemma 3.4. Suppose that $X$ is a topological space such that every two disjoint semiopen and preopen subsets of $X$ can be separated by closed subsets of X. The following conditions are equivalent:

(i) For every two disjoint subsets $G_{1}$ and $G_{2}$ of $X$, where $G_{1}$ is preopen and $G_{2}$ is semi-open, there exists a contra-continuous function $h: X \rightarrow[0,1]$ such that, $h^{-1}(0)=G_{1}\left(\right.$ resp. $\left.h^{-1}(0)=G_{2}\right)$ and $h^{-1}(1)=G_{2}\left(\right.$ resp. $\left.h^{-1}(1)=G_{1}\right)$.

(ii) Every preopen (resp. semi-open) subset of $X$ is a closed subsets of $X$.

(iii) Every preclosed (resp. semi-closed) subset of X is an open subsets of X. 
Proof. (i) $\Rightarrow$ (ii) Suppose that $G$ is a preopen (resp. semi-open) subset of $X$. Since $\varnothing$ is a semi-open (resp. preopen) subset of $X$, by (i) there exists a contra-continuous function $h: X \rightarrow[0,1]$ such that, $h^{-1}(0)=G$. Set $F_{n}=\left\{x \in X: h(x)<\frac{1}{n}\right\}$. Then for every $n \in \mathbb{N}, F_{n}$ is a closed subset of $X$ and $\bigcap_{n=1}^{\infty} F_{n}=\{x \in X: h(x)=0\}=G$.

(ii) $\Rightarrow$ (i) Suppose that $G_{1}$ and $G_{2}$ are two disjoint subsets of $X$, where $G_{1}$ is preopen and $G_{2}$ is semi-open. By Lemma 3.3, there exists a contra-continuous function $f: X \rightarrow[0,1]$ such that, $f^{-1}(0)=G_{1}$ and $f\left(G_{2}\right)=\{1\}$. Set $G=\left\{x \in X: f(x)<\frac{1}{2}\right\}$, $F=\left\{x \in X: f(x)=\frac{1}{2}\right\}$, and $H=\left\{x \in X: f(x)>\frac{1}{2}\right\}$. Then $G \cup F$ and $H \cup F$ are two open subsets of $X$ and $(G \cup F) \cap G_{2}=\varnothing$. By Lemma 3.3, there exists a contracontinuous function $g: X \rightarrow\left[\frac{1}{2}, 1\right]$ such that, $g^{-1}(1)=G_{2}$ and $g(G \cup F)=\left\{\frac{1}{2}\right\}$. Define $h$ by $h(x)=f(x)$ for $x \in G \bigcup F$, and $h(x)=g(x)$ for $x \in H \cup F$. Then $h$ is well-defined and a contra-continuous function, since $(G \cup F) \cap(H \cup F)=F$ and for every $x \in F$ we have $f(x)=g(x)=\frac{1}{2}$. Furthermore, $(G \cup F) \cup(H \cup F)=X$, hence $h$ defined on $X$ and maps to [0, 1]. Also, we have $h^{-1}(0)=G_{1}$ and $h^{-1}(1)=G_{2}$.

(ii) $\Leftrightarrow$ (iii) By De Morgan law and noting that the complement of every open subset of $X$ is a closed subset of $X$ and complement of every closed subset of $X$ is an open subset of $X$, the equivalence is hold.

Corollary 3.5. If for every two disjoint subsets $G_{1}$ and $G_{2}$ of $X$, where $G_{1}$ is preopen (resp. semi-open) and $G_{2}$ is semi-open (resp. preopen), there exists a contracontinuous function $h: X \rightarrow[0,1]$ such that, $h^{-1}(0)=G_{1}$ and $h^{-1}(1)=G_{2}$, then $X$ has the strong cc-insertion property for (cpc, csc) (resp. (csc, cpc)).

Proof. Since for every two disjoint subsets $G_{1}$ and $G_{2}$ of $X$, where $G_{1}$ is preopen (resp. semi-open) and $G_{2}$ is semi-open (resp. preopen), there exists a contra-continuous function $h: X \rightarrow[0,1]$ such that, $h^{-1}(0)=G_{1} \quad$ and $\quad h^{-1}(1)=G_{2}$, define 
$F_{1}=\left\{x \in X: h(x)<\frac{1}{2}\right\}$ and $F_{2}=\left\{x \in X: h(x)>\frac{1}{2}\right\}$. Then $F_{1}$ and $F_{2}$ are two disjoint closed subsets of $X$ that contain $G_{1}$ and $G_{2}$, respectively. Hence by Corollary 3.4, $X$ has the weak $c c$-insertion property for $(c p c, c s c)$ and $(c s c, c p c)$. Now, assume that $g$ and $f$ are functions on $X$ such that $g \leq f, g$ is $c p c$ (resp. $c s c$ ) and $f$ is $c c$. Since $f-g$ is $\quad$ cpc (resp. csc), therefore the lower cut set $A\left(f-g, 2^{-n}\right)=$ $\left\{x \in X:(f-g)(x) \leq 2^{-n}\right\}$ is a preopen (resp. semi-open) subset of $X$. Now setting $H_{n}=\left\{x \in X:(f-g)(x)>2^{-n}\right\}$ for every $n \in \mathbb{N}$, then by Lemma 3.4, $H_{n}$ is an open subset of $X$ and we have $\{x \in X:(f-g)(x)>0\}=\bigcup_{n=1}^{\infty} H_{n}$ and for every $n \in \mathbb{N}, \quad H_{n}$ and $A\left(f-g, 2^{-n}\right)$ are disjoint subsets of $X$. By Lemma 3.2, $H_{n}$ and $A\left(f-g, 2^{-n}\right)$ can be completely separated by contra-continuous functions. Hence by Theorem 2.2, $X$ has the strong $c c$-insertion property for $(c p c, c c)$ (resp. $(c s c, c c))$.

By an analogous argument, we can prove that $X$ has the strong $c c$-insertion property for $(c c, c s c)$ (resp. $(c c, c p c)$ ). Hence, by Theorem 2.3, $X$ has the strong cc-insertion property for $(c p c, c s c)$ (resp. $(c s c, c p c))$.

\section{Acknowledgement}

This research was partially supported by Centre of Excellence for Mathematics (University of Isfahan).

\section{References}

[1] A. Al-Omari and M. S. Md. Noorani, Some properties of contra- $b$-continuous and almost contra-b-continuous functions, European J. Pure. Appl. Math. 2(2) (2009), 213-230.

[2] F. Brooks, Indefinite cut sets for real functions, Amer. Math. Monthly 78 (1971), 10071010. https://doi.org/10.1080/00029890.1971.11992929

[3] M. Caldas and S. Jafari, Some properties of contra- $\beta$-continuous functions, Mem. Fac. Sci. Kochi. Univ. 22 (2001), 19-28.

[4] H. H. Corson and E. Michael, Metrizability of certain countable unions, Illinois J. Math. 8 (1964), 351-360. https://doi.org/10.1215/ijm/1256059678 
[5] J. Dontchev, Characterization of some peculiar topological space via $A$ - and $B$-sets, Acta Math. Hungar. 69(1-2) (1995), 67-71. https://doi.org/10.1007/BF01874608

[6] J. Dontchev, Contra-continuous functions and strongly S-closed space, Internat. J. Math. Math. Sci. 19(2) (1996), 303-310. https://doi.org/10.1155/S0161171296000427

[7] J. Dontchev and H. Maki, On $s g$-closed sets and semi- $\lambda$-closed sets, Questions Answers Gen. Topology 15(2) (1997), 259-266.

[8] E. Ekici, On contra-continuity, Annales Univ. Sci. Bodapest 47 (2004), 127-137.

[9] E. Ekici, New forms of contra-continuity, Carpathian J. Math. 24(1) (2008), 37-45.

[10] A. I. El-Magbrabi, Some properties of contra-continuous mappings, Int. J. General Topol. 3(1-2) (2010), 55-64.

[11] M. Ganster and I. Reilly, A decomposition of continuity, Acta Math. Hungar. 56(3-4) (1990), 299-301. https://doi.org/10.1007/BF01903846

[12] S. Jafari and T. Noiri, Contra-continuous function between topological spaces, Iranian Int. J. Sci. 2 (2001), 153-167.

[13] S. Jafari and T. Noiri, On contra-precontinuous functions, Bull. Malaysian Math. Sc. Soc. 25 (2002), 115-128.

[14] M. Katětov, On real-valued functions in topological spaces, Fund. Math. 38 (1951), 8591.

[15] M. Katětov, Correction to, "On real-valued functions in topological spaces", Fund. Math. 40 (1953), 203-205. https://doi.org/10.4064/fm-40-1-203-205

[16] E. Lane, Insertion of a continuous function, Pacific J. Math. 66 (1976), 181-190. https://doi.org/10.2140/pjm.1976.66.181

[17] E. Lane, PM-normality and the insertion of a continuous function, Pacific J. Math. 82 (1979), 155-162. https://doi.org/10.2140/pjm.1979.82.155

[18] N. Levine, Semi-open sets and semi-continuity in topological space, Amer. Math. Monthly 70 (1963), 36-41. https://doi.org/10.1080/00029890.1963.11990039

[19] S. N. Maheshwari and R. Prasad, On ROs-spaces, Portugal. Math. 34 (1975), 213-217.

[20] H. Maki, Generalized $\Lambda$-sets and the associated closure operator, The Special Issue in Commemoration of Prof. Kazuada IKEDA's Retirement (1986), 139-146.

[21] A. S. Mashhour, M. E. Abd El-Monsef and S. N. El-Deeb, On pre-continuous and weak pre-continuous mappings, Proc. Math. Phys. Soc. Egypt 53 (1982), 47-53. 
[22] M. Mirmiran and B. Naderi, Strong insertion of a contra- $\alpha$-continuous function between two comparable real-valued functions, Earthline J. Math. Sci. 2(1) (2019), 223-239. https://doi.org/10.34198/ejms.2119.223239

[23] M. Mirmiran and B. Naderi, Insertion of a contra- $\alpha$-continuous function, Earthline $J$. Math. Sci. 2(2) (2019), 383-393. https://doi.org/10.34198/ejms.2219.383393

[24] M. Mirmiran and B. Naderi, Insertion of a contra-continuous function between two comparable real-valued functions, Earthline J. Math. Sci. 3(1) (2020), 21-35. https://doi.org/10.34198/ejms.3120.2135

[25] M. Mrsevic, On pairwise $R_{0}$ and pairwise $R_{1}$ bitopological spaces, Bull. Math. Soc. Sci. Math. R. S. Roumanie 30 (1986), 141-145.

[26] A. A. Nasef, Some properties of contra- $\gamma$-continuous functions, Chaos Solitons Fractals 24 (2005), 471-477. https://doi.org/10.1016/j.chaos.2003.10.033

[27] M. Powderly, On insertion of a continuous function, Proceedings of the A.M.S. 81 (1981), 119-120. https://doi.org/10.1090/S0002-9939-1981-0589151-7

[28] M. Przemski, A decomposition of continuity and $\alpha$-continuity, Acta Math. Hungar. 61(12) (1993), 93-98. https://doi.org/10.1007/BF01872101 